\title{
IN VITRO EVALUATION OF MECHANICAL AND STRUCTURAL PROPERTIES OF LEUKOCYTE-PLATELET-RICH FIBRIN AND PLATELET-RICH FIBRIN MATRIX
}

\author{
Reham Lotfy Aggour*, Hadeel A. Sabry** and Gihan Shehatah Hassan***
}

\begin{abstract}
Objectives: The aim of this study was to investigate the mechanical properties as well as the structural analysis of autologous platelet-rich fibrin matrix (PRFM) as compared to autologous leukocyte-platelet-rich fibrin (L-PRF).

Materials\& Methods: Sixty three cubic centimeters (cc) venous blood was obtained from 10 male volunteers. Twenty seven cc were used to prepare 3 specimens of L-PRF (group 1), twenty seven cc were used to get 3 specimens of PRFM (group 2) and nine cc for whole blood analysis. After centrifugation, blood analyses were performed on the residual plasma after collecting L-PRF and PRFM. The L-PRF and PRFM membranes were processed for examination by light microscopy and transmission electron microscopy (TEM) and their mechanical properties were measured by a universal testing machine.
\end{abstract}

Results:Tensile strength and maximum tensile strain of L-PRF group was significantly higher than PRFM group $(\mathrm{p}<0.01)$. PRFM group was significantly stiffer than L-PRF group $(\mathrm{p}<0.01)$. Light microscopy revealed that the platelets were less but more equally distributed in the PRFM than L-PRF. The border between the cellular components and the fibrin network appeared thicker in the PRFM samples than in the L-PRF samples and shows a highly organized network with continuous integrity. TEM showed that both membranes contained two components: a fibrillar material similar to fibrin filaments, and a cellular component that contains human platelet cells. TEM analysis demonstrated that PRFM membrane had more nonactivated platelets.

Conclusion: The present study shows that the structural and mechanical properties of PRFM may fit characteristics desirable for GTR procedures more than that of L-PRF.

KEYWORDS: Mechanical properties, platelet-rich fibrin matrix, leukocyte-platelet-rich fibrin

\footnotetext{
* Lecturer of Oral Medicine, Periodontology, Oral Diagnosis and Oral Radiology, Faculty of Dentistry, October 6 University ** Associate professor of Dental Biomaterials, Faculty of Dentistry, October 6 University

*** Lecturer of Oral Biology, Faculty of Dentistry, Tanta University
} 


\section{INTRODUCTION}

Guided tissue regeneration (GTR) employs a barrier membrane around the periodontal defect to prevent epithelial down growth and fibroblast transgrowth into the wound space, thereby maintaining a space for true periodontal tissue regeneration ${ }^{(1)}$. The GTR membrane which is a cell isolating occlusive biomaterial should meet certain criteria of mechanical, structural and biocompatibility requirements; ability to support organized and vascularized ingrowth; ability to limit the epithelial and unwanted connective tissue growth into the defect; promoting functional tissue regeneration from the relevant cells in the defect; and degrading in adequate time to provide space for newly formed periodontal tissue ${ }^{(2)}$.

Platelet concentrates are classified into four categories; platelet rich plasma (PRP); leukocyte and platelet rich plasma (L-PRP); platelet rich fibrin (PRF) and leukocyte- and platelet rich fibrin (L-PRF). ${ }^{(3)}$

L-PRF was introduced by Dohan et al. as a second-generation platelet concentrate (4). In a simple preparation technique, blood is collected without anticoagulant or thrombin and immediately centrifuged only once. Kawase et al. ${ }^{(5)}$ suggested that the natural fibrin architecture of L-PRF can be used to provide a scaffold for cells and serves as a substrate for the sustained release of growth factors which are the same as those in platelet rich plasma (PRP) ${ }^{(6)}$. L-PRF, in a compressed membrane-like form, has been used as a substitute for commercially available barrier membranes in GTR. However, L-PRF is resorbed within 2 weeks or less at implantation sites, therefore, it can barely maintain adequate space for bone regeneration. The plateletrich fibrin matrix (PRFM) is a variation of PRP and is obtained using a high-speed centrifugation technique, whereby the fibrin matrix is formed by the activation of the clotting cascade by the addition of calcium chloride and a second centrifugation step.
According to Lucarelli et al. PRFM demonstrated robust mechanical properties ${ }^{(7)}$.

Most studies ${ }^{(5,8)}$ on platelet concentrates focus on the platelet and growth factor concentrations and rarely analyze the fibrin structure and the mechanical properties of each product. Nevertheless, the fibrin architecture directly influences the biology of all fibrin-based biomaterials ${ }^{(9)}$ and mechanical characteristics of the membrane may affect the final results of GTR ${ }^{(10)}$. Thus, further investigation is necessary to look further into all features of these biomaterials. This will enable us to comprehend the clinical results obtained and subsequently extend the fields of their therapeutic application.

Some authors ${ }^{(11)}$ examined structural analysis of L-PRF. However, to our knowledge, no study has been conducted for a structural analysis of PRFM. The main objective of this study was to examine the composition, mechanical properties and structural characteristics of PRFM. The secondary objective was to compare these characteristics to those of L-PRF.

\section{MATERIALS \& METHODS}

Blood samples were collected at the Department of Oral Medicine and Periodontology, Faculty of Dentistry, October 6 University (Egypt) in September 2015 from 10 healthy male volunteers (age range: 22 to 30 years; mean age: 27 years) with no history of smoking, systemic diseases or intake of medications that might interfere with coagulation over the previous 3 months. After obtaining an informed consent, the blood samples ( 7 tubes of $9 \mathrm{ml}$ each) were obtained from antecubital vein of each volunteer. Four tubes contained an anticoagulant for whole blood analysis (1 tube) and PRFM (3 tubes) production. The 3 other tubes contained no anticoagulant for L-PRF production. The study was conducted in accordance with the Helsinki Declaration of 1975, as revised in 2000. 


\section{Preparation of L-PRF}

L-PRF was prepared according to the technique described by Dohan et al. ${ }^{(3)}$. Blood samples were collected in 10-mL tubes without an anticoagulant. The tubes were immediately centrifuged at 400 $\mathrm{g}$ for $10 \mathrm{~min}$ in a table centrifuge (Centurion, Scientific limited, UK), at room temperature, following which the blood in the tubes separated into 3 layers: the RBC layer that occupied the lower part of the tube, the L-PRF layer in the middle of the tube and acellular plasma at the top of the tube. The L-PRF was thus removed from the tube using sterile tweezers, separated from the RBC base using scissors and pressed between two pieces of surgical gauze to obtain the L-PRF membrane.

\section{Preparation of PRFM}

Twenty- seven mL of human blood were collected into three sterile $10-\mathrm{mL}$ blood collection tubes. After gentle mixing, the tubes were centrifuged at $1100 \mathrm{~g}$ for 6 minutes to obtain PRP. PRP was transferred into calcium chloride-containing tubes $(0.25 \mathrm{~mL}$ $\mathrm{CaCl} 2 \mathrm{1M}$ ) under sterile conditions. The tubes were gently swirled and then immediately placed in a table centrifuge (Centurion, Scientific limited, UK) and centrifuged at $4500 \mathrm{~g}$ for 25 minutes at room temperature. A translucent, yellow-white plateletfibrin matrix, having the same inner diameter as the tube was recovered at the bottom ${ }^{(8)}$.

\section{Blood Parameters Measurement}

Platelet counts were performed on the donors' whole blood and on the residual serum remaining after L-PRF and PRFM preparation with a Haematology analyzer (Beckman Coulter Inc., Brea, CA, USA). Fibrinogen concentration was measured on the same samples.

\section{Mechanical Testing}

The membranes were cut into $10 \mathrm{~mm} \times 5 \mathrm{~mm}$ specimens in accordance to ASTMD 1708. All specimens were stored in sterile saline solution at $4^{\circ} \mathrm{C}$ and tested after five days from preparation. All tests were performed at $25^{\circ} \mathrm{C}$.

Tensile strength $(S)$, tensile strain $(\varepsilon)$, stiffness (elastic modulus $E$ ) and toughness of the specimens were evaluated using a universal testing machine (Instron 3345, Scientific Services, USA) (Figure1). Tensile loading of $10 \mathrm{~N}$ was applied at a cross head speed of $2 \mathrm{~mm} / \mathrm{min}$. The maximum load at specimen failure was recorded and tensile strength was calculated using the formula $S=F / A$ as $F$ is the maximum applied load $(\mathrm{N})$ and $A$ is the unit area $\left(\mathrm{m}^{2}\right)$. Stress-strain curve was recorded with LE instron Bluehill software. Stiffness of the specimens was calculated from the curve and the total area under the curve demonstrated the toughness of the specimens. Particular attention was paid to fix the sample on the holder of the testing station. Elastomeric tags were used to achieve secure grip of every specimen.

\section{Preparation for Light-Microscopy}

L-PRF and PRFM membranes were dehydrated in increasing concentrations of alcohol (70\%, 95\%, and $100 \%$ ) and placed in two grades of xylene before paraffin inclusion. For each membrane, a series of successive $7-\mu \mathrm{m}$ sections were performed according to the long axis of the membrane. These sections were stained using Hematoxylin and Eosin.

\section{Preparation for Transmission Electron Micro- scope}

L-PRF and PRFM membranes were fixed with a glutaraldehyde $2.5 \%$ then fixed with osmium tetroxide $2 \%$ buffered. Subsequently, the membranes were dehydrated in successive passes of ethanol at concentrations of 50\%, 70\%, 85\%, 95\% and $100 \%$. Finally, they were placed in acetone. Then, the membranes were embedded in a mixture of 1:1 epoxy propane and EPON for 2 hours and delivered to the capsule in a $40^{\circ} \mathrm{C}$ oven for 1 hour and a $60^{\circ} \mathrm{C}$ oven for 48 hours. The polymerized samples were used to obtain ultrathin sections of 
approximately $60 \mathrm{~nm}$. The samples were stained for contrast with uranyl acetate and lead citrate. The sections were examined with JEOL transmission electron microscopy (JEOL JEM-100SX) ${ }^{(12)}$.

\section{Statistical Analysis}

The statistical analysis was performed using a commercial software programme (SPSS 14.0, SPSS Inc., Chicago, IL, USA). Data were analyzed with Mann-Whitney $\mu$ test for intergroup comparisons. $P$ values less than 0.05 were considered statistically significant.

\section{RESULTS}

\section{Blood Parameter Measurements}

Analysis of the residual serum showed that no detectable platelets were left after L-PRF membrane extraction, indicating that almost all platelets were incorporated in the L-PRF (Table 1). The residual serum remained after obtaining PRFM showed that only $1,500 \pm 515.52$ of platelets were left in the serum denoting that about $99.5 \%$ of the platelets were present in the PRFM. Additionally, results shown in table (1) demonstrate that, after preparation of L-PRF and PRFM, no detectable fibrinogen was left in the residual serum. These results indicate that all the fibrinogen present within the blood samples was polymerized to fibrin in the L-PRF and PRFM.

\section{Mechanical properties}

The results are summarized in table (2). Tensile strength and maximum tensile strain of L-PRF group was significantly higher than PRFM group ( $p$ $<0.000)$. PRFM was significantly stiffer than L-PRF $(p<0.000)$. Additionally, toughness of L-PRF was significantly higher than reported for PRFM ( $p=$ 0.031).

TABLE (1) Fibrinogen and platelet concentration in whole blood and residual serum remained after leukocyte-platelet-rich fibrin and platelet-rich fibrin matrix preparation. Data are presented as mean \pm standard deviation $(n=10)$

\begin{tabular}{|l|l|l|l|l|}
\hline & \multicolumn{2}{|c|}{ Platelet Rich Fibrin } & \multicolumn{2}{c|}{ Platelet Rich Fibrin Matrix } \\
\hline Platelet $\left(10^{3} / \mathbf{m m 3}\right)$ & $\begin{array}{l}\text { Whole Blood } \\
319 \pm 81,146\end{array}$ & $\begin{array}{l}\text { Residual Serum } \\
\text { Undetectable }\end{array}$ & $\begin{array}{l}\text { Whole Blood } \\
290,50 \pm 50,516\end{array}$ & $\begin{array}{l}\text { Residual Serum } \\
1,5 \pm 5.16^{\#}\end{array}$ \\
\hline $\begin{array}{l}\text { Fibrinogen concentration } \\
(\mathbf{m g} / \mathbf{d L})\end{array}$ & $\begin{array}{l}\text { Whole Blood } \\
355 \pm 55.1\end{array}$ & $\begin{array}{l}\text { Residual Serum } \\
\text { Undetectable }\end{array}$ & Whole Blood & Residual Serum \\
\hline
\end{tabular}

TABLE (2) Mechanical characteristics of leukocyte-platelet-rich fibrin and platelet-rich fibrin matrix. Data are expressed as mean \pm standard deviation.

\begin{tabular}{|l|l|l|c|}
\hline Measured values & PRF & PRFM & $P$ value \\
\hline Tensile Strength $(\mathbf{M P a})$ & $0.11045 \pm 0.06$ & $0.0852 \pm 0.07$ & 0.000 \\
\hline Maximum Tensile Strain $\mathbf{( m m / m m )}$ & $1.4456 \pm 0.12$ & $0.72634 \pm 0.09$ & 0.000 \\
\hline Elastic Modulus $(\mathbf{M P a})$ & $0.0764 \pm 0.07$ & $0.1173 \pm 0.09$ & 0.000 \\
\hline Toughness $\left(\right.$ joule $\left./ \mathbf{m}^{3}\right)$ & $3.8418 \pm 0.63$ & $3.5256 \pm 0.52$ & 0.031 \\
\hline
\end{tabular}

MPa = Megapascal 


\section{Light-Microscopic Study:}

Macroscopically, three parts could be distinguished in the L-PRF clot. A yellow portion which represented the main body, a small peripheral red portion and a whitish layer between them which was called the "buffy coat". Microscopically, a cell-free dense fibrin network was found to predominate in the yellow portion of the L-PRF. Also, most of the cellular components (white blood cells and platelet aggregates) were entrapped mostly in the buffy coat. In the lower red portion, RBCs were entrapped within a fibrin matrix with few cellular components. Certainly, the cytoplasm of the leukocytes and RBCs were stained dark pink. Unfortunately, the dark blue/violet stain of the platelet aggregates and leukocyte's nuclei made the distinction between them was only possible by morphological examination in the microscope and therefore was dependent on the observer. (Figures 2a, 2b).

On the other hand, macroscopically, the PRFM appeared as a translucent yellow white disk with nearly similar two sides. Microscopically, the cellular components were less in PRFM than in L-PRF. Also, dense fibrin fibrils encircled the membrane and intervened inside it; forming a more organized network with a continuous integrity. The boundary of this network appeared thicker. Moreover, Platelets were found in groups in the margins of this network separated by thick fibrils. This network was also surrounded by thin fibrin fibrils with few platelets. (Figures 3a, 3b).
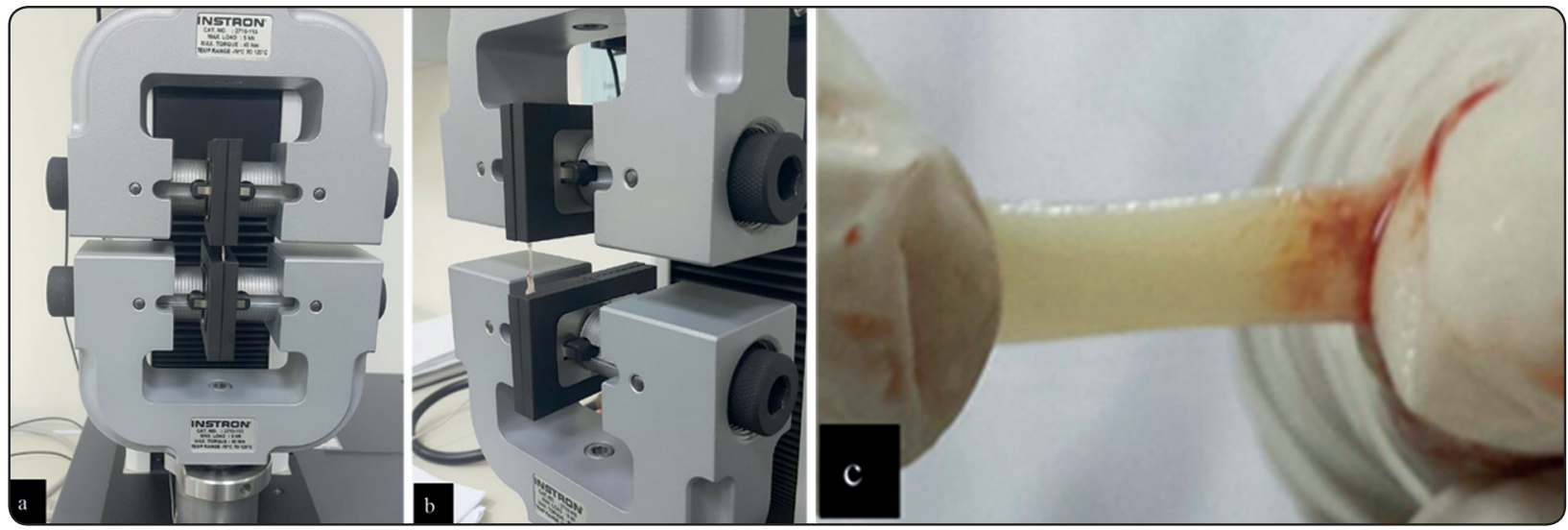

Fig. (1) Testing the mechanical properties of PRFM.



Fig. (2) Light micrograph of the L-PRF clot showing (a): fibrin network (A), the buffy coat portion (B) and the red blood cell portion (C). (b) Higher magnification of the square area illustrating that fibrin appeared homogeneous in light pink. The leukocytes and platelets nuclei were more localized at the junction between the RBCs and the fibrin portion. Few leukocytes could be easily identified (arrows). a: (H\&E. X400), b: (H\&E. X1000). 


\section{Transmission electron microscope evaluation}

TEM was used to describe the ultrastructure architecture of the PRF and PRFM membranes. In the PRF membrane, the platelets showed signs of activation.As some platelets displayed degranulation and extension of pseudopodia. In addition, partial rupture of the platelet membrane could be observed in some platelets. Also, intact granules within the cytoplasm and extracellular environment beside fibrin fibrils were evident (Figure 4a). Whereas, the PRFM membrane demonstrated many nonactivated platelets. Most of the platelets displayed discoid appearance. Moreover, the platelet-specific granules
( $\alpha$-granules and dense bodies) showed peripheral arrangement (Figure 4b).

\section{DISCUSSION}

Collagen based materials have shown favorable results in GTR procedures due to excellent biocompatibility, however, there is product variability and lack of control over the resorption rate which is a concern for GTR. Moreover, the use of membranes derived from animal tissues is associated with the risk of disease transmission and has raised certain ethical and cultural issues ${ }^{(13,}$ ${ }^{14)}$. It has been shown that fibrin-based membranes
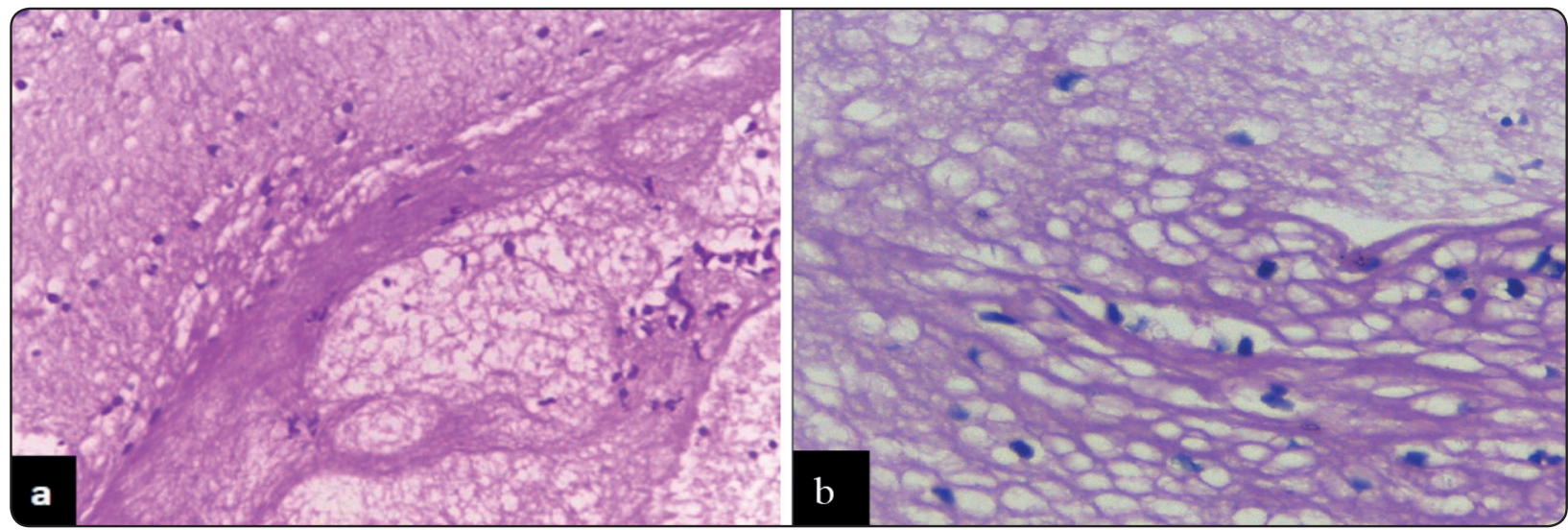

Fig. (3) Light micrographs of the PRFM demonstrates (a): a highly organized network with continuous integrity. Notice the cellular components were less than in the PRF. (b): The border between the cellular components and the fibrin network appeared thicker. a: (H\&E. X400), b: (H\&E. X1000).
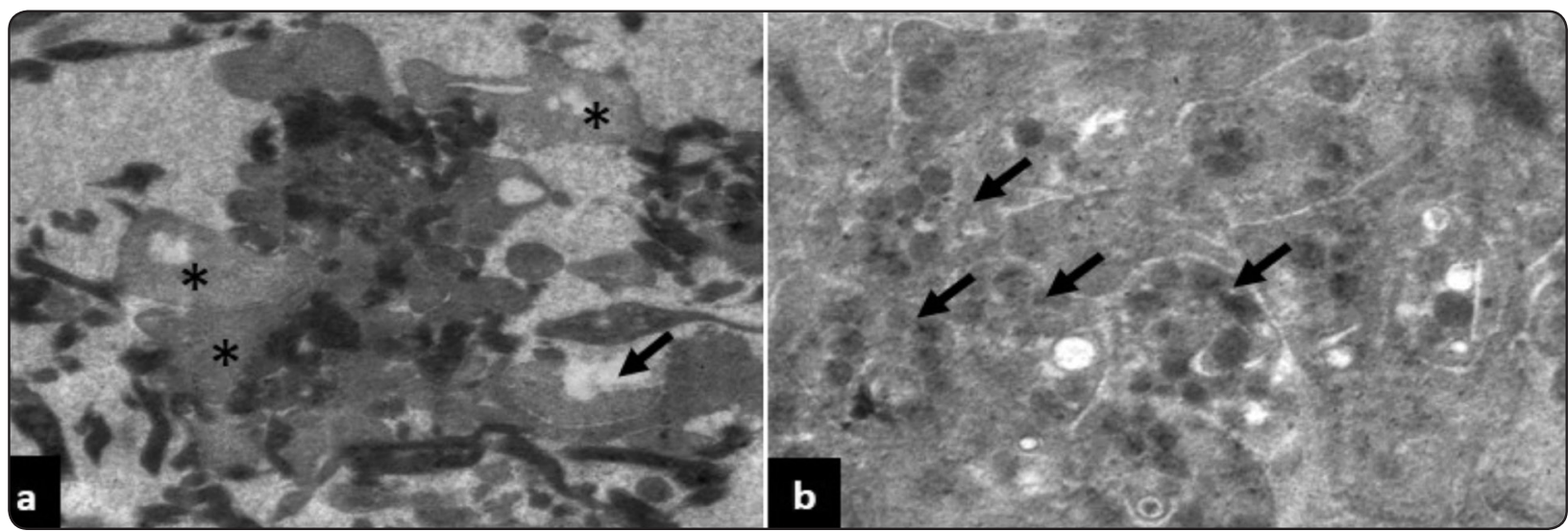

Fig. (4) Transmission electron micrographs of the membranes showing (a): the PRF membrane shows active platelets with complete loss of the granules $(*)$ and ruptured platelet (arrow). (b): the PRFM membrane shows many nonactive platelets with peripheral distribution of the granules (arrows). a, b (3000x) 
could be better scaffolds for proliferation of periosteal and osseous cells when compared to collagen membranes in vitro ${ }^{(15,16)}$. However, to use fibrin-based membranes in GTR applications, the thorough assessment of all characteristics of these membranes is required before its clinical use.

This study was designed to examine the structural and mechanical characteristics of two platelet concentrates; L-PRF and PRFM. Fibrin architecture, ultrastructure and mechanical properties are important parameters for ideal scaffold material (17). Strong mechanical characteristics of a scaffold provide more suitable support for regeneration ${ }^{(18)}$.

The results showed that the tensile strength, the maximum tensile strain and the toughness of L-PRF were significantly higher than PRFM though the stiffness of PRFM was significantly higher. These results may be due to their structural differences which may be partially attributed to their differences in polymerization. The mode of polymerization has significant effects on mechanical properties of fibrin matrix ${ }^{(19)}$. Moreover, the increased elastic modulus of PRFM when compared to L-PRF indicates that the stiffness of an autologous platelet-fibrin preparation can be increased by inducing extensive fibrin network formation through increased gravitational force during centrifugation. The increased elastic modulus of PRFM confers more pliability when compared to L-PRF however increased tensile strength of L-PRF allows the membrane to closely conform to a variety of irregular surgical sites more than PRFM.

Our results are contradictory to the results obtained by Khorshidi et al (20) who compared L-PRF versus PRGF/Endoret membrane. Although calcium chloride is used in PRGF Endoret system, the second centrifugation used in PRFM preparation isn't used. In PRGF/Endoret, calcium chloride is used to initiate the last coagulation stage; then sudden fibrin polymerization occurs ${ }^{(20)}$. Therefore the fibrin matrix is immature and most of the fibrils are thin ${ }^{(9)}$. On the other hand, the slow and natural polymerization that occurs during the centrifugation of PRF produces fibrin fibrillae assembled in equilateral junctions which provides a flexible and elastic fibrin network unlike the week bilateral junctions found in PRGF/Endoret (9). Additionally, the circulating fibrinogen present in L-PRF and PRFM strengthens the final fibrin matrix ${ }^{(9)}$. Alston et al. ${ }^{(21)}$ and Duong et al. ${ }^{(22)}$ indicated that increase in fibrinogen concentration makes the final fibrin matrix stronger. Another important difference between these two membranes is the presence of more quantities of leukocytes in L-PRF than that in PRFM. Some authors suggest that removing leukocytes from platelet-rich concentrates may prevent their potential negative effects on the extracellular matrix caused by anti-inflammatory effects of proteases and hydrolase ${ }^{(23,24)}$. However, the negative effects of leukocytes on fibrin matrix are controversial ${ }^{(25)}$.

Typically applied to engineering materials, especially when in the shape of sheets, tensile tests have been used as a first approach to get information about the mechanical performance of the membranes (26). Performing tensile tests is easy and related results are often easy to manage. The preference for one characteristic or the other depends on the technical application of the material. Due to the autologous nature of L-PRF as well as PRFM, their physical properties are less than reported with commercially available collagen membranes.

Transmission electron microscopic (TEM) examination was performed to study the ultrastructure of L-PRF and PRFM. Both membranes contain a fibrillar and a cellular (largely human platelet cell) component. This unique structure may be capable of acting as a matrix for carrying of cells that are essential for periodontal regeneration. However, the clusters of platelets seen along the PRFM demonstrate more complete and regular membrane which indicates that the platelets 
are in an inactive state (resting) which will be transformed into the activated state, along with the sustained release of growth factors, in the healing site. Light microscopy of PRFM showed that the fibrin strands were thicker with more branching and more organized architecture when compared to L-PRF. However, these favorable characteristics are not evident through the entire membrane as some fibres are deformed compared to their natural shape. This arrangement could contribute to the increased elastic modulus. More studies are needed to clarify the relationship between centrifugal force and duration applied to various platelet concentrates and mechanical properties of resulting fibrin -based membranes. The 25 minute centrifugation at $4500 \mathrm{~g}$, at $25^{\circ} \mathrm{C}$, needed to prepare the PRFM may not be enough for fibrin synthesis and crosslinking to go to completion.

The membranes that are used in GTR should have certain mechanical and structural properties to protect initial blood clot and regenerative process. The present study shows that the production of PRFM made through high-speed centrifugation of intact platelets and fibrin in the absence of exogenous thrombin yields a membrane with characteristics desirable for guided tissue regeneration (GTR) procedures.

\section{REFERENCES}

1. Gottlow J. Guided tissue regeneration using bioresorbable and non-resorbable devices: Initial healing and long-term results. J Periodontol 1993; 64:1157-1165.

2. Hughes F.J., Ghuman, M. Tall A. Periodontal regeneration: a challenge for the tissue engineer. Proc Inst. Mech. Eng. H 2010; 224: 1345-1358.

3. Dohan Ehrenfest DM, Rasmusson L, Albrektsson T. Classification of platelet concentrates: From pure plateletrich plasma (P-PRP) to leucocyte- and platelet- rich fibrin (L-PRF). Trends Biotechnol 2009; 27: 158-167.

4. Dohan, D.M., Choukroun, J., Diss, A., et al. Platelet-rich fibrin (PRF): a second-generation platelet concentrate. Part I: technological concepts and evolution. Oral Surg Oral Med Oral Pathol Oral Radiol Endod 2006; 101: 37.
5. Kawase, T., Okuda, K., Saito, Y., and Yoshie, H. In vitro evidence that the biological effects of platelet-rich plasma on periodontal ligament cells is not mediated solely by constituent transforming-growth factor-beta or plateletderived growth factor. J Periodontol 2005; 76: 760.

6. Dohan Ehrenfest DM, de Peppo GM, Doglioli P, Sammartino G. Slow release of growth factors and thrombospondin-1 in Choukroun's platelet-rich fibrin (PRF): A gold standard to achieve for all surgical platelet concentrates technologies. Growth Factors 2009; 27:63-69.

7. Lucarelli E, Beretta R, Dozza B, Tazzari PL, O’Connell SM, Ricci F, Pierini M Squarzoni S, Pagliaro PP, Oprita EI, and Donati D. A recently developed bifacial platelet-rich fibrin matrix. Eur J Cells Materials. 2010; 20:13-23.

8. Castillo TN, Pouliot MA, Kim HJ, Dragoo JL Comparison of growth factor and platelet concentration from commercial platelet-rich plasma separation systems. Am J Sports Med 2011; 39(2):266-271.

9. Buchta C, Hedrich HC, Macher M, Hocker P, Redl H. Biochemical characterization of autologous fibrin sealants produced by CryoSeal and Vivostat in comparison to the homologous fibrin sealant product Tissucol/ Tisseel. Biomaterials 2005; 26:6233-6241.

10. Lee SB, J.-S. Kwon, Y.-K. Lee, K.-M. Kim, and K.-N. Kim, "Bioactivity and mechanical properties of collagen composite membranes reinforced by chitosan and tricalcium phosphate," Journal of BiomedicalMaterials Research Part B: Applied Biomaterials, 2012; 100: 19351942.

11. David M. Dohan Ehrenfest, Marco Del Corso, Antoine Diss, Jaafar Mouhyi and Jean-Baptiste Charrier,. ThreeDimensional Architecture and Cell Composition of a Choukroun's Platelet-Rich Fibrin Clot and Membrane. J Periodontol 2010; 81:546-555.

12. Silva et al.: Ultrastructural characteristics of fibrin clots from canine and feline platelet concentrates activated with calcium gluconate or calcium gluconate plus batroxobin. BMC Veterinary Research 2013; 9:77.

13. Dash M., F. Chiellini, R.M. Ottenbrite, E. Chiellini, Chitosan-a versatile semisynthetic polymer in biomedical applications, Prog. Polym. Sci. 2011: 36; 981- 1014.

14. Schwartzmann M, Use of collagen membranes for guided bone regeneration: a review, Implant Dent. 2000: 9: 63-66. 
15. Gassling V, T. Douglas, P. H. Warnke, Y. Ac, il, J. Wiltfang, and S. T. Becker, "Platelet-rich fibrin membranes as scaffolds for periosteal tissue engineering," Clinical Oral Implants Research, 2010; 21: 543-549.

16. Gassling V, J. Hedderich, Y. Ac,il, N. Purcz, J. Wiltfang, and T. Douglas, "Comparison of platelet rich fibrin and collagen as osteoblast-seeded scaffolds for bone tissue engineering applications," Clinical Oral Implants Research, 2013: 24: 320-328.

17. Zhang Y, X. Zhang, B. Shi, and R. Miron, "Membranes for guided tissue and bone regeneration," Annals of Oral \& Maxillofacial Surgery, 2013: 1: 10.

18. Lynch SE, Tissue Engineering: Applications in Oral and Maxillofacial Surgery and Periodontics, Quintessence Publishing Company, 2008.

19. Kumar RVand N. Shubhashini, "Platelet rich fibrin: a new paradigm in periodontal regeneration," Cell and Tissue Banking, 2013; 14: 453-463.

20. Hooman Khorshidi, Saeed Raoofi, Rafat Bagheri, and Hodasadat Banihashemi, "Comparison of the Mechanical Properties of Early Leukocyte- and Platelet-Rich Fibrin versus PRGF/Endoret Membranes," International Journal of Dentistry. 2016; 2016.
21. Alston SM, K.A. Solen, A. H. Broderick, S. Sukavaneshvar, and S. F. Mohammad, "New method to prepare autologous fibrin glue on demand," Translational Research, 2007;149: 187-195.

22. Duong H, B.Wu, and B. Tawil, "Modulation of 3Dfibrin matrix stiffness by intrinsic fibrinogen-thrombin compositions and by extrinsic cellular activity," Tissue Engineering - Part A 2009; 15: 1865-1876.

23. Anitua E, M. S'anchez, G. Orive, and I. Andia. Delivering growth factors for therapeutics. Trends in Pharmacological Sciences, 2008; 29: 37-41.

24. Anitua E, M. S'anchez, G. Orive, and I. And'1a, "The potential impact of the preparation rich in growth factors (PRGF) in different medical fields," Biomaterials, 2007; 28: 4551-4560.

25. Ehrenfest DMD, L. Rasmusson, and T. Albrektsson, "Classification of platelet concentrates: from pure plateletrich plasma (P-PRP) to leucocyte- and platelet-rich fibrin (L-PRF)," Trends in Biotechnology, 2009; 27: 158-167.

26. Alvarez V, Mondragn I, V zquez A. Influence of chemical treatments on the interfacial adhesion between sisal fibre and different biodegradable polymers. Compos Interfaces 2007; 14(7-9):605-616. 